\title{
Learning and Change Networks
}

\author{
Jean Annan and Rose Carpenter
}

\begin{abstract}
The Learning and Change Network Strategy (LCN) was developed to raise students' achievement by appreciating, extending and enriching their learning environments. Throughout New Zealand there are 53 voluntary, networked communities of practice. They involve the active participation of students, teachers, parents, school leaders and community members from early childhood centres and primary, intermediate, secondary, and special education schools. Together the participants explore learning environments in relation to mutually-understood achievement challenges and make changes based on unique, contextually-specific analyses. Networks are observing impacts of this change on student outcomes and the practice of all participants. They have noticed that many children have made academic gains, collaboration within and between schools and communities has increased, and teachers, parents and students themselves have gained new insights into the students' learning. Schools have benefited from the broadening of leadership and evaluative capability of staff and the transfer of in-depth methods of inquiry into school initiatives.
\end{abstract}

\section{Practice Paper}

Keywords: change, collaboration, learning environments, school networks

\section{LEARNING AND CHANGE NETWORKS}

When I reflect on previous interventions, they were 'done to' students with minimal lines of communication between leader, teacher, student, and home. The sharing of this information with other schools in our community has changed our focus from 'our kids' to 'our communities' kids'. Parents are now far more comfortable engaging in conversation about their kids' learning. It has developed a stronger rapport with parents, school and child, and is having other positive spin-offs around how the parents want to participate in school programmes. There is greater collegial support amongst local schools than previously (Network Leader).

\section{The Learning and Change Strategy}

The Learning and Change Strategy (LCN) is an organic, contextually-responsive approach to educational change in New Zealand. This cuttingedge initiative, recognised internationally through its contribution to the OECD's Innovative Learning Environments project (ILE), has been designed to raise student achievement by extending learning connections and aligning student, family, whānau and school perspectives on current and future education. It has focused particularly on the learning supports of those students who have yet to reach literacy and numeracy expectations. The LCN strategy has grown collaboration within and between schools and communities. Schools throughout the country have committed time to learning what will make a difference for their students' achievement and have channelled new energy into addressing the longstanding challenge of successfully supporting the learning of those students whose academic scores regularly occupy the bottom levels.

LCN is a three-way partnership developed in 2011 among the New Zealand Ministry of Education, UniServices-Faculty of Education, the University of Auckland and the Networks (see Annan \& Talbot, 2013). Specifically, the establishment of the networks for learning was expected to promote the following goals:

- Equitable outcomes for students who are Māori or Pasifika or who have special educational needs

- Educational environments that are responsive to students' learning paths

- Active participation of students, teachers, family/ whānau and community in learning activity

- New knowledge built upon the expertise existing within networks and communities

- The creation of mechanisms for knowledge transfer across and beyond networks 
- Enhancement of leadership and evaluative capability at network level and within networks' schools

- System-wide improvement through the collaborative growth of learning systems.

\section{New Roles in a New World}

LCN corresponds with global interest in developing learning environments for the rapidly changing, diverse and increasingly connected world. This development calls for new perspectives on learning and teaching, new tools and new practices. Many students can now, through the internet and other means, readily establish multiple and diverse learning relationships and quickly access a vast amount of information. They have the means and capability to assume an active role in interpreting and elaborating their curricula. These students are learning how to learn and where to access the information they require for their current and future learning journeys. Learning is viewed as an exciting, on-going process in which the students are actively engaged.

If relevant educational experience is to be available for all students, modes of teaching and learning must be designed to support culturally-responsive and future-focused learning (Fullan, 2013; Hampson, Patton \& Shanks, 2012; Hannon, 2009). Contemporary pedagogies encourage teachers, parents and students to embrace the uncertainty, pace and inevitability of current and future learning environments. LCN attempts to open up such opportunities to greater numbers of students. The networks create opportunities for interactive participation to build on participant knowledge, expertise and resources to change systems and practices that impact on the educational outcomes of students. In-depth exploration of students' broad learning environments within networks of practice ensures that strategies for change are carefully considered, not haphazard, imposed or restricted by unquestioning adherence to familiar tools. Decisions are based on practice-based evidence, next steps emerging from contextually-specific and globallyinformed analyses conducted within the LCN networks. These actions are built into the LCN process.

New roles necessarily involve changes in practice for everyone involved with students' learning and the revision of personal and professional identities. For teachers, this involves carefully constructing unique and contextually-relevant plans for professional development, derived from the change priorities of the network rather than prescribed menus with which many have become accustomed. Clearly, this process cannot take place over-night. Accordingly, Learning and Change Networks have not rushed the change but have engaged in deep, collaborative explorations and analyses of students' learning contexts and activity over several months. They have taken time to ensure that students, teachers, whānau and families make direct contributions to understandings reached, plans for change and indicators of progress. The LCN activities and processes that have supported this collaborative work are discussed later in this article.

\section{Situated Learning in Communities of Practice}

Networks have been established to make a positive difference. As networks are not effective per se, and can be counter-productive and insular if not set up to succeed (Wenger, 2010a), they have been facilitated in ways that foster strong communities, shared understandings and common practices. This included activity that promoted collective valuing of the purpose of the network. This was achieved through shared identification of the achievement challenge and visual representation of students' learning environments. It also involved systematic analysis, conducted through collaborative, iterative content analysis that reduced large amounts of data to manageable emerging themes. Common practices were developed through shared but personalised planning that was guided by the shared analysis. Facilitation of network-to-network interaction fostered the establishment and maintenance of connections inside and outside of the network, allowing for the formation of contextually-relevant knowledge.

LCN networks have been configured on the concept of Community of Practice, a structure that holds knowledge and learning at its core (Wenger, 2010b). With it roots planted firmly in ecological and situated learning theory, LCN assumes a broad, connected and dynamic notion of learning (Lave \& Wenger, 1991; Wenger, McDermott \& Snyder, 2002). From this perspective, understandings of the current and future learning environments necessarily take into account the unique experiences of learners and the social nature of learning. Creating new learning environments involves those who are significant in students' learning and does not rely solely on replicating practices from high-performing schools or from other projects. Home-school partnerships and the exchange of cultural knowledge are vital if learning environments are to support the learning of all students (Bull, Brooking \& Campbell, 2008; Dumont, Istance \& Beavides, 2010; Fullan, 2013; Hannon, 2009; The NZ Ministry of Education, 2010).

Communities of Practice have been formed by networked schools and serve as vehicles for knowledge exchange and creation. They strive to ensure that students, and adults, have the range of tools, access to knowledge, flexibility and 
agency they need in their current and future lives. Communities of Practice are characterised by their shared concern for a particular, dynamic body of knowledge, voluntary participation, balance of commonality and diversity within the membership, equivalent value of the contributions of all participants, shared power and connected learning activity. Accordingly, in LCN activity, the balance between knowledge provision and facilitation of co-directed learning is constantly shifting as LCN networks develop cohesion.

\section{Learning and Change Networks}

There are currently $53 \mathrm{LCN}$ networks of school communities ranging in size from 3 to 14 schools. The total number of networks comprise seven early childhood centres, 19 kura, 285 primary schools, 25 intermediate schools, 30 secondary schools and 14 special education schools. Participation by schools, individuals and networks is entirely voluntary.

Student-learning is paramount in the Learning and Change Strategy. Networks comprise students, parents and whānau, teachers and school leaders, iwi and other key community groups. These participant groups come together to appreciate students' broad learning environments and identify platforms on which to build student achievement. Together, participants learn about the conditions that support engagement in learning activity and shape the nature and direction of next steps. Each participant takes an active role in designing, implementing and reviewing change activities and developments. They periodically assess the extent to which their own practice changes in network-determined priority areas have impacted on students' connection with learning and academic achievement.

Within LCN networks there are three key leadership roles; principals, teacher-enthusiasts, and evaluators, who in most, but not all cases, are teachers in networking schools. The principals link the strategy to their schools' governance, strategic resources and provide support as required while, in most cases, the teacher-enthusiast drives the initiative in the school. Generally, there are two or three evaluators in the network depending on the size of the group. Some networks have chosen to have an evaluator in each school. Evaluators take a lead role in ensuring that systems are in place to measure students' learning and the changes in participant practice.

A feature of the LCN Strategy has been the new opportunities LCN networks have taken to exchange and create knowledge with other networks (Figure 1). Change has been initiated through dialogue and debate created through participants' common concern for student-learning and the diverse perspectives represented in each LCN network. Change in teaching and learning practice has been informed by the shared and valued knowledge within the network, information purposely sought from outside the network, and new knowledge created through the synthesis of knowledge from various sources.



Figure 1. Networks of Learning and Change networks.

Learning and Change networks are facilitated to encourage the following:

- The establishment and development of lateral learning connections for all participants

- Appreciation of positive supports and achievements in developmental contexts

- Active participation and collaboration of all participants

- The cultural alignment of students' various learning environments.

Facilitators support networks to negotiate a balance of structured and unstructured change-activity to achieve their goals. In English medium schools, networks are, in the early stages, facilitated directly by the University of Auckland implementation team and the Ministry of Education support and advice team. In Māori-medium kura, the facilitation team works with local iwi and Māori education organisation leaders who make decisions about the way LCN is conducted in their kura. One network, Kura-a-Iwi, has a direct relationship with the Ministry of Education with regard to LCN.

Facilitation has been available for a finite period and for a specific purpose: that is, to support schools to form LCN networks and establish their early tasks. When networks reach the point at which they are self-sustaining, external facilitation - that is, facilitation provided by the University of Auckland is gradually phased out and network members assume 
this role. The nature of LCN network activity changes over time as networks develop. In the facilitated development phase, network schools are most likely to synchronise their activity as they establish new practices. The schedules of subsequent cycles of learning what to change and what is changing are more likely to respond to real-time events in schools and communities with the nature of support among members becoming more diverse, timely and particularised. In strong LCN networks, this is a highenergy phase.

\section{LEARNING AND CHANGE NETWORK ACTIVITY}

LCN networks have, over the past 24 months, worked through the Learning and Change Framework (Figure 2). At this point, most networks, other than those that are newly-formed, are implementing changes. The following section provides a brief summary of the broad activity of LCN networks.

\section{The Achievement Challenge}

At the outset, participants in each network meet to identify suitable, mutually-understood and valued student-achievement challenges. These become the focus of network activity. Networks have chosen to understand their most challenging achievement areas, the most frequently selected achievement challenges in current learning and change networks involving writing (33 percent), reading (20 percent), or mathematics (12 percent). Several networks have chosen to consider students' achievement in more general areas such as literacy or academic achievement across multiple subjects (UniServices-Faculty of Education, 2014). Networks also discuss and clarify the roles of participants and plan initial network activities.

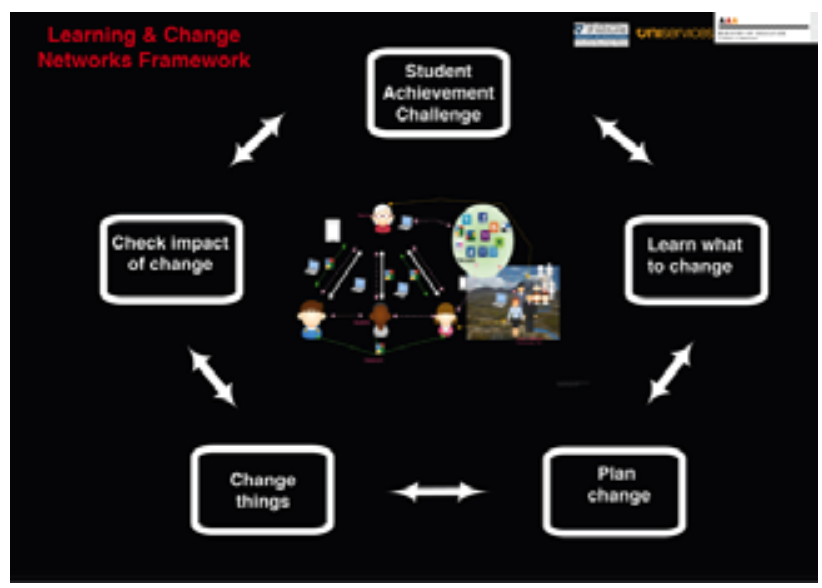

Figure 2. The Learning and Change Network Framework.

\section{Learn What to Change}

With a clear view of the achievement challenge, LCN networks learn what practices, structures or tools to change in order to improve students' achievement. Learning what to change involves examining the students' learning environments and analysing findings to identify the various influences, particularly supports, on achievement. Plans for exploring students' learning environments are designed within networks and are based on participants' early hunches about what impacts on the achievement challenge. Some LCN networks have selected or developed their own means to investigate, but most have chosen to begin with Learning Environment Maps. Learning Environment Maps are created by students who illustrate by drawing or arranging items to show their sites of learning, the tools and artefacts associated with learning, the people who help them learn, and the connections among these. Learning Environment Maps are created in many ways including paper and pen, computer programmes, or arrangement of cut-out figures. For students who do not communicate in verbal language or for whom drawing or arranging is not possible, learning maps may be created with those who know the students best. Discussion around the maps provides the context for an Investigation of Practice in which students, parents and teachers consider their own current actions and the ways in which each might contribute to the enhancement of the students' futurefocused learning environments.

The in-depth information analysis is appreciative, systematic and collaborative, and has been facilitated by the Learning and Change team from the University of Auckland. Networks analysed the contents of the information gathered between network meetings and noted the dominant themes emerging from the data. For some networks, explorations and analyses have been iterative processes of interpreting and elaborating practices. For others, multiple data sets have been analysed simultaneously at the end of the 'learning what to change', also called the 'understanding' stage. For all students, networks have identified the supports and strengths that serve as pillars to support new practices. Once networks view the emerging themes as being distinctly formed and reaching saturation, they are re-labelled as 'priorities for change'. These priorities for change guide subsequent planning.

Analyses of students' broad learning environments result in the identification of priority areas of change. The most common change priorities in the current LCN networks are:

- Establishing learning-focused family and community connections

- Facilitating student agency and the active participation of students 
- Student engagement

- Lateral learning

- Digital technology in schools and communities.

\section{Planning Change, Changing, and Checking for Impact}

Change activities are designed by all participants together and represent the different roles each takes in pursuing achievement challenges. Participants also determine the indicators of progress. Changes in practice are informed by different sets of knowledge. These are:

- Knowledge from inside the network (e.g. collective, existing knowledge within the network of practice, information from students' learning maps)

- Knowledge from outside the network (e.g. from professional and academic research, other networks).

- New knowledge created by the group (i.e. synthesis of information from inside and outside of the LCN network.

LCN networks purposefully and specifically construct change activities driven by coherent sets of principles derived from their information analysis. These infiltrate the broadening learning environment and influence the nature of everyday interaction between students and others. They may lead to specified activities such as tailored professional development or the use of digital platforms.

Networks monitor progress in terms of:

a. Changes in practices in priority areas

b. Movement toward the achievement challenge.

Some LCN networks have selected to create their own change-in-practice criteria so that their evaluative measures align with their identified change priorities. Others have chosen to conduct standard surveys, established within the LCN Strategy, to measure change in pre-specified areas related to the Ministry of Education's benefit outcomes. As the strategy has, in this initiative, been focused on raising student achievement, National Standards and Ngā Whanaketanga Rumaki Māori information has been requested for Year 1 to Year 8 students as a way of tracking progress toward the achievement challenge.

\section{CHANGES NOTICED BY PARTICIPANTS}

Information about changes in learning and practice has been shared by networks through Milestone Reports 3, 4 and 5 to the Ministry of Education and videos taken in network environments. Changes have been observed in students' learning, the practices that influence this learning, participant relationships and the on-going reflection on, and refinement of, network activity. LCN networks became more aware of the value of data-driven decision-making and appreciation of the role of qualitative information in understanding that which is quantitative. Listed below are some of the changes noticed by participants.

\section{Academic Learning}

Changes in academic achievement were observed for the years 2012/13 and 2013/14. For 1112 students, whose achievement data were analysed to compare end-of-year 2012 and end-of-year 2013 achievement, there was an observed increase in the number achieving at or above National Standards (Milestone 3 Report). All schools that forwarded National Standards information had participated in the project for the entire year. As available data included that from only 53 of the 155 schools, no overall conclusions can be drawn from this initial result. The total number of schools included secondary schools that did not have National Standards data as well as special schools for which this measure was not suitable for tracking progress. However, what is of interest in this observation is that some students made significant progress even though they had been involved only in the Learning What to Change phase of the strategy. It appeared that, for these students, change had occurred concurrently with their involvement in the exploration and understanding of their learning environments. This is not a surprising observation, a basic premise of the appreciative inquiry threading through the LCN strategy being that understanding and change occur at the same time.

Data were collected to examine National Standards' performance for participants between end-of-year 2013 and end-of-year 2014. A comparison was made between data for this period from 3795 students in 178 English-medium schools - Māori-medium schools' data being analysed separately because Ngā Whanaketanga Rumaki Māori standards were used. The results of the analysis of National Standards data showed an overall positive 24.43 percent point change, constituting a mean quartile shift for this cohort. Although the majority of students in the LCN strategy had been initially achieving below National Standards, the ratings at the end of 2014 for 927 of these students were at or above National Standards. Shifts were greatest in the areas of writing and mathematics, areas commonly selected by groups of schools as foci for their network activity (UniServicesFaculty of Education 2014 Milestone 5 Report).

\section{Students' Positive Views of Learning}


Students have commented on the way LCN has helped them connect with learning and sharing their work on an ongoing basis. One student commented on the way technology has allowed him to share his work with parents:

We've got heaps more technology. We always used to write in our books and our parents didn't see what we were doing until we brought our books home, at the end of the year, pretty much. And this year, we can email our work to mums and dads.

Many students have also demonstrated greater confidence in approaching learning tasks. One student, initially reluctant to talk about learning, discussed her newfound confidence:

I think I'm quite a confident learner. Like, I would put myself out there if I didn't know the answer. If I wanted to say something I would put my hand up, not holding back my ideas, cause I could be right or I could be wrong, and sometimes it doesn't matter about the answer, as long as you're trying.

From the outset, students have taken active roles in sharing information, making interpretations of their learning environments and designing changes. Active involvement in learning has been observed at school and students are increasingly working alongside adults at network meetings. One student commented that "If you are an active learner, then you think for yourself, but if you aren't an active learner, then ... someone thinks for you". Newer networks, with the benefit of knowledge provided by earlier networks, are including students and parents in the full range of network activity much earlier in their formation.

\section{New Insights into Students' Learning}

Several LCN network leaders have commented that they have gained insights into their students' learning that had not surfaced through routine methods of exploring learning environments. Participants of networks had worked to create strong links between settings to promote coherence across the students' multiple learning environments. A network leader commented that this had "sparked a desire to work collaboratively with other schools to connect and engage with our families" and to develop culturallyresponsive learning environments.

\section{Lateral Learning: New Teaching and Learning Relationships and Insights into Learning Environments}

Groups of students, parents, teachers and school leaders have come together, bound by their common interest in the students' learning. During the network formation period, some LCN networks, particularly those who had worked together previously, quickly developed trusting relationships. Some had, in the early stages, worked to resolve tensions associated with new processes, such as the relative benefits of pursuing a particular achievement challenge for each school, differences in view or even a history of competition among participants. As participants have come to know one another better, the interaction within these networks has become increasingly collaborative and interdependent. In many LCN networks, the largely flat structure has become a mechanism for creating and sharing knowledge across the broad contexts of students' learning. Increased trust among network members has led to authentic collaboration. Many networks are working together to develop and implement across-network moderation processes involving all of their teachers, not only a small group of leaders. In one school for example, writing samples were taken from classrooms across each school and then all schools' samples were considered across the network and in another. Most schools involved in LCN networks have made visits to other schools in their networks, the visiting teams generally involving students, teachers, parents/ whānau. During these visits, network members have exchanged ideas about learning and developed new learning relationships.

Parents have indicated they value exchanging ideas between school and home. Here is one example:
I really want my kids to have their culture instilled in them. That's why that partnership that we talk about - the teachers and parent - I believe the teacher also needs to understand the cultural background of the child, because children will learn better if those things are being respected as well, at school, so it's like vice versa. Like I respect what the teacher's trying to do in her job, and support, and that's exactly what I would expect from the teacher as well.

Many LCN networks have commented on the value of working with other schools, linking through site visits and other 'between meeting' activity. One network reported that " [Participation in the LCN] has brought the local schools closer together in discussions on the important things - students learning and improved achievement". Networks experienced greater trust amongst schools and now appreciated a biggerpicture view of the educational pathways of their students. The value of peer interaction along this path was clear as noted by a school leader:

The power of children learning from children ... has been highlighted through this process. 
At times networks have been challenged by the geographical isolation of their schools. Many have found ways to connect with one another, either through travelling for face-to-face meetings and visits or through digital means. One and two teacher school leaders have commented on the value of forming communities of practice through LCN networking that link them with one another and with larger schools. The LCN has also provided opportunities for networks to connect with other networks through regional networking days. In some areas, participants were welcoming new schools into their networks.

\section{Customised Change Activities}

Networks have commented on the critical role of allowing them the autonomy to ask open questions and to respond to their unique contexts. One network leader commented:

We are enjoying the level of autonomy and shared accountability of the network ... it allows the network to be a living, changing and developing learning network of professionals. We are accountable to our learners and community. A positive aspect is shared leadership. We've built a shared language. Trust has been built. Great potential of vertical network - early childhood through to secondary school. This way of working is a model for what we want for our students. Breakdown in the competitiveness of schools and building of true collaborations.

Knowledge of what to change in order to meet achievement-challenges and how to make changes has emerged from the collaborative engagement of all participants who have collected and analysed data. Priority changes, designed to meet the achievementchallenge, have not been prescribed by the Ministry of Education advisors, the facilitation team, or the LCN network leaders. Each network is unique, its structure and activity negotiated within the network to suit the particular context. Networks have not sought a one-size-fits-all approach to learning what to change or planning and implementing change. They have not sought off-the-peg programmes but implemented those that have been purposefully built on knowledge inside and outside of the network.

The LCN approach to understanding students' learning environments has provided schools the opportunity to identify areas for tailored teacher professional development: for example, to prepare LCN networks for contemporary and future-focused learning, some networks have chosen to learn more about supporting learning through the use of digital technology, perhaps using particular platforms such as Google Classrooms.

\section{Broadening of Leadership and Evaluative Capability Through Schools and Networks}

Networks have reported improved use of data to understand students' learning. Most networks providing information for Milestone Report 3 commented on the role of systematic, data-informed inquiry in learning what to change and how to make changes. One network leader commented that: "Data has driven every aspect ... of initial planning". Networks have observed that greater value is now placed on qualitative data to make sense of broad quantitative data.

In many LCN networks the broadening distribution of leadership has been evident. Practitioner evaluators, some of whom have not held leadership positions in their schools, are now taking a lead role in observing changes, analysing data and communicating findings.

\section{Transfer of the Learning and Change Approach to Other School and Network Initiatives}

Schools report that some of their core systems, such as school planning and reporting, and the development of students' individual plans, have been influenced by their participation in the Learning and Change Strategy. Several have embedded processes for in-depth understanding into everyday practice and, in some cases, the involvement of students in decisions about them is now business-as-usual. Several networks commented that the methodology for creating change through open exploration and active participation of all stakeholders has also been explicitly applied in other new projects in their schools. One network leader commented on the impact of LCN on school systems:

We plan to use the LCN frame as a full system shift that has an impact on our students.

Some networks were concerned about the time required for participation in LCN in the early stages of the strategy, particularly when they were connecting with other schools and gathering the ecological data that allowed them to articulate change priorities. However, as multi-participant practices have become more integrated into schools' everyday routines, and active learning and teaching have become an assumed part of classroom activity rather than additional to existing practices, for some, the process has become less demanding.

\section{FURTHER OBSERVATIONS}

The following links provide pathways to more information about the Learning and Change Strategy process: 
- Education Gazette: Learning and Change Networks: Connecting students with learning http://www.edgazette.govt.nz/Articles/Article. aspx?Articleld $=9016$

- Rose Patterson: The New Zealand Initiative. 'No school is an island. Fostering collaboration in a competitive system http://nzinitiative.org.nz/site/nzinitiative/files/ publications/publications\%202014/NSIAI_Web_ FINAL1.pdf

- Jenny Jackson (School Principal): Blog http://jennyljackson.blogspot.it/2014/04/now-thati-have-got-my-principals-blog.html

- Chris Theobald (School Principal): Cartoon description of Learning and Change https://www.youtube.com/watch?v=IZ895_WMQ3Y

- Learning and Change video clips: https://www.youtube.com/user/LCNNZ/playlists

\section{NEW QUESTIONS AND NEXT STEPS}

While many networks have reported considerable change in the way that learning and teaching occur in their schools and communities, the Learning and Change Strategy has, for some, raised as many questions as it has answered. The questions raised provide networks with information about their next steps. What has been established is a forum for discussions around these questions. For example, networks are working to decide what authentic and constructive community involvement means in their particular context. They ask "How can we support involvement with our community?" The increasingly active role taken by students may not be something anticipated by parents, or for some teachers or students. Similarly, expectations of developing unique locally and globally relevant professional development plans may not have been an expectation for all involved. Collaboration among schools and communities requires time to develop. It involves understanding strongly-held beliefs and perspectives that have been developed through a lifetime of first-hand experience.

LCN networks have also contemplated the nature of measurement for their priority changes as the need to complement traditional metrics has become apparent. They are seeking and constructing indicators of change that involve active learners in interactive environments and are sensitive to changes in critical qualities and experiences that are not easily quantified or objectively observed. Networks are also working to ensure that their practices spread beyond 'pockets of promise' to support all students in their schools.

\section{SUMMARY}

If all students are to benefit from the educational opportunities available in the current and future worlds and be prepared to negotiate their futures, new learning environments that support engagement and achievement of each student are required. Although opportunities for active connection with new modes of learning may present naturally for some young people, there is no guarantee that such options will be open to every student.

The Learning and Change Network Strategy is a threeway partnership, created specifically to consider and develop new learning environments for those students who are already challenged to achieve at expected levels. Participation is entirely voluntary and characterised by an appreciative, positive approach to thinking and talking about students' learning pathways. LCN networks of schools have opened up opportunities for 6500 students, their families and schools that have participated in the strategy.

LCN networks have developed context-specific change strategies and new practices as a result of systematically conducting open and collaborative explorations of students' current and preferred learning environments. The Community of Practice model on which the networks have been established has supported interaction through a balance of commonality and diversity, and a mutual focus on a specified achievement challenge. It has provided a structure that encourages exchange of cultural knowledge between home/community and school. Participants have taken time to understand the multiple perspectives on learning of the various member groups, avoiding knee-jerk reactions to identified challenges to learning. They have worked over a period of several months to ensure that all parties have had authentic opportunities to contribute to the collective understanding and that the underlying influences, rather than surface effects, are understood.

Numerous benefits of participation have been expressed by LCN networks. In many of these deliberately constructed Communities of Practice there are examples of new lateral learning relationships among all participants, distribution of leadership across networks, appreciation of students' contexts of learning, a balance of structure and informality, and data-supported decision-making. 


\section{REFERENCES}

Annan, B., \& Talbot, J. (2013). International conference on innovative learning environments. New Zealand system note. Retrieved from https://cdn.auckland.ac.nz/assets/education/about/ learning-change-networks/innovativelearning.pdf

Bull, A., Brooking, K., \& Campbell, R. (2008). Successful home-school partnerships. Report to the New Zealand Ministry of Education. Wellington: Ministry of Education.

Dumont, H., Istance, D., \& Beavides, F. (Eds.) (2010). The nature of learning. Practitioner guide from the innovation learning environments project. Centre for Educational Research and Innovation.

Fullan, M. (2013). Stratosphere: Integrating technology, pedagogy, and change knowledge. Toronto, Canada: Pearson Canada.

Hampson, M., Patton, A., \& Shanks, L. (2012). Ten ideas for $21^{\text {st }}$ century education. London: Innovation Unit.

Hannon, V. (2009). The search for next practice. A $U K$ approach to innovation in schools. Education Canada, 49(4), 24-27.

Lave, J., \& Wenger, E. (1991). Situated learning: Legitimate peripheral participation. Cambridge: Cambridge University Press.

The New Zealand Ministry of Education (2010). Effective governance: Working in partnership. Retrieved from http://www.minedu.govt.nz/ /media/ MinEdu/Files/Boards/EffectiveGovernance/ WorkingInPartnership.pdf

The New Zealand Ministry of Education (2013). The Māori education strategy: Ka hikitia - accelerating success, 2013-2017, Retrieved from http://www. minedu.govt.nz/theMinistry/PolicyAndStrategy/ KaHikitia.aspx

The New Zealand Ministry of Education (2013). The Pasifika education plan. Retrieved September 25, 2014 from http://www.minedu.govt.nz/NZEducation/ EducationPolicies/PasifikaEducation/ PasifikaEducationPlan2013.aspx
UniServices-Faculty of Education, The University of Auckland (2014, February). Learning and change milestone report 3. Report to the New Zealand Ministry of Education. Retrieved from https:/cdn.auckland.ac.nz/assets/education/about/ learning-change-networks/LCN\%20Milestone\%20 Report\%203\%20.pdf

UniServices-Faculty of Education, The University of Auckland (2014, July). Learning and change milestone report 4. Report to the New Zealand Ministry of Education. Retrieved from https://cdn.auckland.ac.nz/assets/education/about/ learning-change-networks/Milestone\%204\%20 report\%20final.pdf

UniServices-Faculty of Education, The University of Auckland (2014, February). Learning and change milestone report 5. Report to the New Zealand Ministry of Education. Retrieved from https://cdn.auckland.ac.nz/assets/education/about/ learning-change-networks/LCN\%20MS5\%20 Final\%20revised $\% 20$ to $\% 20$ client $\% 2012$ Feb\%20 15.pdf

Wenger, E. (2010a). Communities of practice and social learning systems: The career of a concept. In C. Blackmore (Ed.), Social Learning Systems and Communities of Practice (pp. 179-198). London: The Open University - Springer-Verlag.

Wenger, E. (2010b). Conceptual tools for CoPs as social learning systems. In C. Blackmore (Ed.), Social learning systems and communities of Practice (pp. 125-144). London: The Open University -Springer-Verlag.

Wenger, E., McDermott, R., \& Snyder, W. (2002). Cultivating communities of practice: A guide to managing knowledge. Boston, MA: Harvard Business Press. 


\section{AUTHOR PROFILES}



Jean Annan (PhD) works at UniServices-Faculty of Education, University of Auckland where she is the Strategy Development Advisor for the Learning and Change Network project. She is an educational psychologist whose particular interests lie in working with the ecological systems that impact on students' development, well-being and connections with learning.

\section{Email:}

jean@positively.co.nz

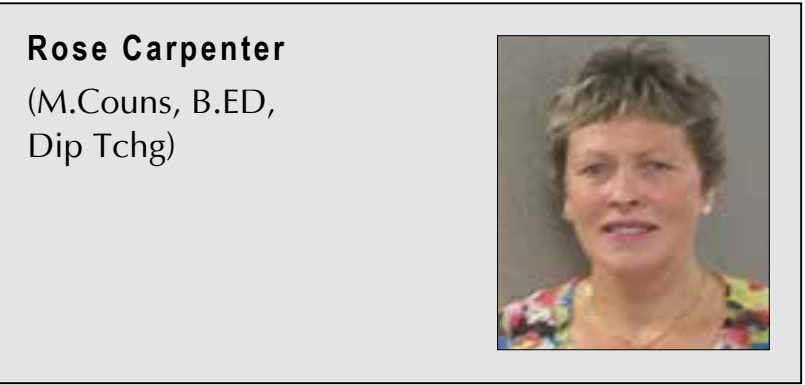

Ko Takitimu to waka

Ko Ngati Kahungungu rāua ko Ngai Tahu ngā iwi

Ko Ruahine to maunga

Ko Makaretu te awa

Ko Rakautatahi te marae

Rose Carpenter works at the Ministry of Education, Wellington where she is the National Manager for the Learning and Change Networks and Mutukaroa projects. She is passionate about raising student achievement levels with a priority focus on achievement levels of Māori learners, Pasifika learners, learners from low socio-economic backgrounds and learners with special needs by developing student agency and family/whānau agency. Rose is an advocate for listening to the 'stories of others' in addressing equity issues as well as working collaboratively to make a genuine difference in and for the lives of others.

\section{Email:}

R.Carpenter@education.govt.nz 